\title{
Worksharing Tasks: an Efficient Way to Exploit Irregular and Fine-Grained Loop Parallelism
}

\author{
$1^{\text {st }}$ Marcos Maroñas \\ $2^{\text {nd }}$ Kevin Sala \\ $3^{\text {rd }}$ Sergi Mateo \\ Barcelona Supercomputing Center (BSC) Barcelona Supercomputing Center (BSC) Barcelona Supercomputing Center (BSC) \\ marcos.maronas@bsc.es \\ kevin.sala@bsc.es \\ sergi.mateo@bsc.es
}

\author{
$4^{\text {th }}$ Eduard Ayguadé \\ Barcelona Supercomputing Center (BSC) \\ Universitat Politècnica de Catalunya (UPC) \\ eduard.ayguade@bsc.es
}

\author{
$5^{\text {th }}$ Vicenç Beltran \\ Barcelona Supercomputing Center (BSC) \\ vicenc.beltran@bsc.es
}

\begin{abstract}
Shared memory programming models usually provide worksharing and task constructs. The former relies on the efficient fork-join execution model to exploit structured parallelism; while the latter relies on fine-grained synchronization among tasks and a flexible data-flow execution model to exploit dynamic, irregular, and nested parallelism. On applications that show both structured and unstructured parallelism, both worksharing and task constructs can be combined. However, it is difficult to mix both execution models without penalizing the data-flow execution model. Hence, on many applications structured parallelism is also exploited using tasks to leverage the full benefits of a pure data-flow execution model. However, task creation and management might introduce a non-negligible overhead that prevents the efficient exploitation of fine-grained structured parallelism, especially on many-core processors. In this work, we propose worksharing tasks. These are tasks that internally leverage worksharing techniques to exploit fine-grained structured loop-based parallelism. The evaluation shows promising results on several benchmarks and platforms.
\end{abstract}

\section{INTRODUCTION}

The introduction of the first multiprocessor architectures led to the development of shared memory programming models. One of those is OpenMP, which became a de facto standard for parallelization on shared memory environments.

OpenMP [1], with its highly optimized fork-join execution model, is a good choice to exploit structured parallelism, especially when the number of cores is small. Worksharing constructs, like the well-known omp for construct, are good examples of how OpenMP can efficiently exploit structured parallelism. However, when the number of cores increase and the work distribution is not perfectly balanced, the rigid forkjoin execution model can hinder performance.

The omp for construct accepts different scheduling policies that can mitigate load-balancing issues; and the nowait clause avoids the implicit barrier at the end of an omp for. Still, both techniques are only effective in a few specific situations. Moreover, the fork-join execution model is not wellsuited for exploiting irregular, dynamic, or nested parallelism.

Task-based programming models were developed to overcome some of the above-mentioned limitations. The first tasking models were based solely on the tasks and taskwaits prim- itives, which naturally support irregular, dynamic, and nested parallelism. However, these tasking models are still based on the fork-join execution model. The big step forward came with the introduction of data dependences. Thus, replacing the rigid fork-join execution model by a more flexible data-flow execution model that relies on fine-grained synchronizations among tasks. Modern task-based programming models such as as Cilk, OmpSs or OpenMP tasking model have evolved with advanced features to exploit nested parallelism [2], hardware accelerators [3][4][5], and seamless integration with message passing APIs such as MPI [6].

The flexibility of the data-flow execution model relies on the dynamic management of data-dependences among tasks. However, dependences management might introduce a nonnegligible overhead depending on the granularity and number of tasks. Hence, finding the adequate task granularity becomes a key point to get good performance: too many fine-grained tasks will increase task overheads, but too few coarse-grained tasks will hinder the available parallelism. Yet, it is not always possible to reach the optimal granularity that is coarse enough to compensate for the overheads while opening sufficient parallelism. Moreover, the granularity is limited by the problem size per core. Thus, if the problem size per core is too small, the granularity might be suboptimal, hurting the performance.

For those situations, it makes sense to combine both strategies - tasking and worksharing - in a way that we can palliate the drawbacks of each strategy while maximizing their strengths. To do so, we propose an extension to tasks especially adapted to avoid the fork-join execution model. We introduce the for clause applied to the task construct in the OmpSs-2 programming model [7].

Our evaluation shows promising results. The task for construct enlarges the set of granularities where peak performance is reached. Additionally, it enables users to increase peak performance by up to $10 \mathrm{x}$ compared to pure task-based implementation for small problem sizes with low parallelism.

To sum up, the contributions of this paper are (1) a proposal and implementation of worksharing tasks, which is a mechanism combining tasking and worksharing parallelization 
strategies; and (2) an evaluation of such anto implementation on several benchmarks and two different architectures.

The rest of this document is structured as follows: Section III contains the motivations behind this work; Section III reviews the most relevant related work; Section IV explains the changes affecting the model; Section V details our implementation; Section VI consists of an evaluation and discussion of the proposal; Section VII summarizes the work done and provides concluding remarks; and, finally, Section VIII presents future work proposals.

\section{Motivation}

Fine-grained loop parallelism can be found in most HPC applications. So, it is important to develop techniques that perform well for this kind of parallelism. Harris et al. [8], already explored the importance of properly supporting finegrained loop parallelism.

Nowadays, developers can use loop-based parallelism or task-based parallelism for coding their applications containing fine-grained loop parallelism. Loop-based parallelism is quite simple to write, and it performs well in architectures with a low number of cores and applications with a small load imbalance. Despite this, it implies a rigid synchronization resulting in performance drops when facing many-core architectures and imbalanced applications. Task-based parallelism allows a dataflow execution, which is more flexible than its loop-based counterpart. Additionally, it provides several key benefits, previously mentioned in Section [1. Thus, it usually performs well in many-core architectures and load imbalanced applications.

Still, an inherent problem of task programming is granularity choice. If task granularity is not adequately set, overhead may penalize overall performance. The overhead of tasks is caused by several different sources. The first one is the actual task creation, which usually implies costly dynamic memory allocations. Secondly, the computation of dependences between tasks, which involves the use of dynamic and irregular data-structures. Finally, the scheduling of the tasks across many cores can also become a bottleneck.

Task granularity and the number of created tasks are inversely proportional. Consequently, a given problem can be solved either by using many fine-grained tasks or a few coarsegrained ones. Thus, finding an adequate granularity is a key point to optimally exploit resources when using tasks [9], alleviating the aforementioned overheads, but still creating enough parallelism to maximize resource utilization.

A typical granularity chart is shown in Figure 1 . The $\mathrm{x}$ axis varies the granularity of tasks, while the y-axis represents performance. The chart presents the results of the synthetic benchmark shown in Code 1. There are three different series representing different problem sizes. The chart also contains coloured parts which represent different chart (not application) phases. Note that from $X=256$ to $X=1 K$, phases 1 and 3 are merged. This is because it is Phase 1 for $P S=16 \mathrm{~K}$, but Phase 3 for $\mathrm{PS}=128 \mathrm{~K}$ and $\mathrm{PS}=1 \mathrm{M}$.

When the problem size is $1 \mathrm{M}$ and $128 \mathrm{~K}$, there are three well-differentiated phases. In the first phase, we can see how

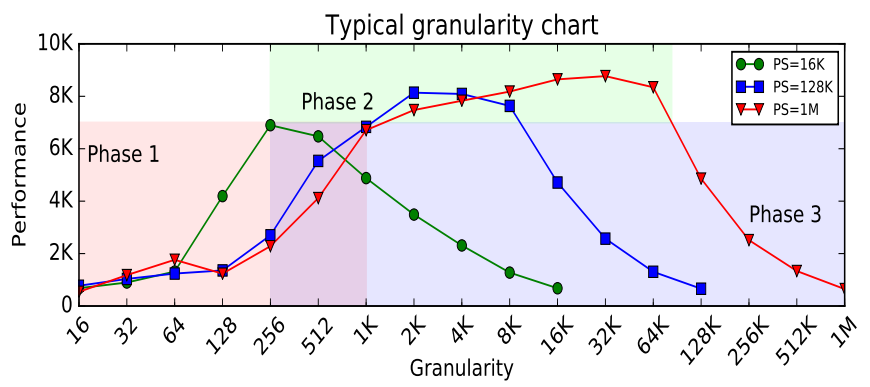

Fig. 1: Typical granularity chart.

the performance is low because there are too many very finegrained tasks and the overheads of creation and management of that amount of small tasks are too costly. In the second phase, performance grows until reaching peak performance. Finally, in the third phase, performance decreases again because there is not enough parallelism (i.e., not enough tasks are being created to feed all the CPUs).

\section{Code 1: OMP F}

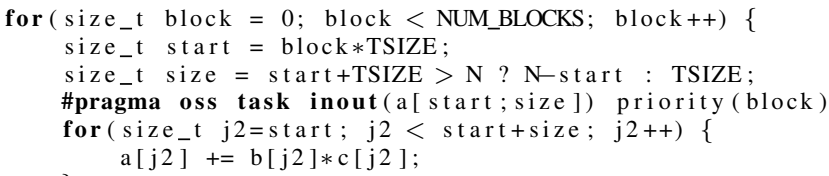

Typically, a good granularity allows having, at least, one work unit per core to occupy all the resources. Ideally, having more than one work unit per core is better to prevent some load balance problems. Additionally, it is important setting a granularity coarse enough to alleviate task management overheads. However, there is a crucial factor that can limit the granularity choice: the problem size per core. The problem size per core is the result of dividing the total problem size by the number of available cores. In consequence, the problem size per core only depends on the total problem size and the number of cores available in our system.

In an ideal case, like the problem sizes of $1 \mathrm{M}$ and $128 \mathrm{~K}$ in Figure 1, the granularity can grow until the overhead is not a problem. At that point, the problem size per core is big enough to create sufficient tasks - of a granularity that is not affected by the overhead - to feed all the resources. This happens in the second phase when peak performance is reached.

In contrast, if the problem size per core is not big enough, the developer must decide between a finer granularity that is still affected by the overhead but creates sufficient parallelism, or a coarser-granularity that is less affected by the overhead but causes a lack of parallelism. When this happens, the second phase of the typical granularity chart does not appear, being unable to reach peak performance. This phenomenon occurs in Figure 1 when the problem size is $16 \mathrm{~K}$.

Daily, developers are involved in situations where the problem size per core is not optimal, jeopardizing the use of tasks in their applications.

1) Strong scaling in distributed environments: This is a common scenario in HPC environments. Strong scaling starts 
from a given problem size per core, and make it smaller either by augmenting the number of resources or by decreasing the total problem size. As we have seen, reducing the problem size per core while maintaining the granularity of the tasks can lead to insufficient work.

2) Many-core architectures: Increasingly, architectures have more and more cores. This trend directly affects the problem size per core, which becomes reduced because the same problem size is divided among more resources. Thus, setting an adequate granularity becomes harder or even impossible, leading us to either increased overhead or lack of parallelism.

3) Applications requiring different granularities: Many applications rely on different kernels to perform a computation, and each of them may require a different task granularity to achieve optimal performance. Finding an adequate granularity that fits all the different algorithms may be impossible. For this case, it is especially important to have a broad set of granularities where peak, or at least acceptable, performance is reached because if all the kernels have several granularities that reach peak performance, it is easier to find a granularity that performs well for all the kernels than it would be if there is a single granularity getting peak performance for each kernel.

Additionally, it may happen that an application with different kernels must share the same granularity. The reason is that the data partitioning may implicitly set the task granularity. When this happens, it is especially important having a wide set of granularities performing well in all the kernels. This way, it is easier to find a coincidence across all the sets.

Apart from this, granularity issues may prevent runtime libraries from developing sophitiscated and smart policies. Those policies may introduce few overhead per task but could provide benefits in terms of programmability and performance. However, if a program contains a huge number of tasks, the aforementioned small overhead per task, rapidly becomes unaffordable. A good example is the support for region dependences. This kind of dependences enables users to annotate their codes with the whole memory regions a task actually access. Then, the runtime library computes the dependences with all the partial overlappings, actually preventing any task that shares even a single byte to execute until the current task finishes. In Code 2, using region dependences, the second task depends on the first task, while it does not when using discrete dependences (e.g., OpenMP dependences) because those only consider the start address. Region dependences are very useful to simplify codes, but they come at a cost. The computation of the dependences is more expensive compared to discrete dependencies. In consequence, if the number of tasks is huge, the overhead may become excessive.

Code 2: Region deps

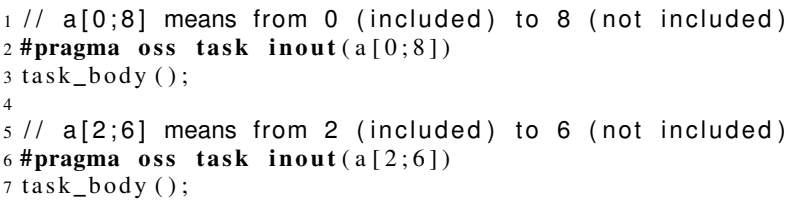

To sum up, task-based parallelism offers several key benefits that developers want to keep. Notwithstanding, there are currently several difficulties or problems when programming fine-grained loop parallelism with tasks. (1) Granularity is critical: for that purpose, a thorough and time-costly analysis must be done in order to choose it adequately; (2) adequate granularity does not always exist: some scenarios may force developers to choose either overhead or lack of parallelism; and (3) runtime libraries cannot develop sophisticated tasking management policies: those could jeopardize the performance in programs with a very large task number.

\section{RELATED WORK}

Our proposal, based on the idea of hierarchical partitioning, is broadly used in distributed environments to reduce overheads. Most applications firstly partition data using inter-node parallelism, spreading such data among different nodes. Then, the work is partitioned again using intra-node parallelism. There are several works in the literature proposing several techniques based on this idea, such as [10], [11], and [12].

As OpenMP is the standard for shared memory parallelism, we performed a thorough review of the OpenMP environment to search related work. This can be seen in Section III-A In addition, wider related work can be found in Section III-B

\section{A. OpenMP related work}

On OpenMP, this hierarchical partition of data can be implemented in several ways using a combination of worksharing and tasking constructs.

One of those is using tasks to perform a first partition of the work, and then, each task contains a nested parallel region with a worksharing construct. The reason for using tasks in the first level of partitioning is the flexibility given by the data dependences. This implementation may increase the resource utilisation in some scenarios, boosting performance. However, we end up introducing a barrier inside each task, at the end of the nested parallel region.

Barriers have been broadly treated in literature [13]. Currently, they are usually highly optimized so that they introduce only a few overhead in some situations, though, if the work is not perfectly balanced, the intrinsic rigidity of the forkjoin model may lead to undesired waiting times. OpenMP introduced the nowait clause to palliate this issue. This clause omits the barrier at the end of a worksharing region. Nonetheless, this mechanism is not useful to avoid the barrier at the end of a parallel region inside a task. This is because the barrier is necessary to postpone the release of the task dependences until the work is completed.

OpenMP also provides different scheduling policies for the worksharing constructs, alleviating load imbalance problems. Still, they are not enough for many cases, and the rigidity of the fork-join model may lead to an underutilization of the resources.

It is possible to implement a different solution using task nesting. This is basically creating tasks inside tasks. So, a 
first partitioning is done using coarse-grained tasks with data dependences, which are then partitioned into fine-grained tasks without data dependences. The second level of partitioning reduces the overhead compared to a single level of partitioning where all the tasks have data dependences because the nested tasks do not pay the dependence management costs. In addition, using tasks improves load balance. However, tasking introduces other overheads associated with tasks management, such as dynamic allocations and task scheduling.

The OpenMP tasking model also provides the taskloop construct. Applied to a loop, it partitions the iteration space by generating several tasks. There is the possibility of specifying a grainsize guaranteeing that each of the tasks created executes no less than grainsize iterations. With this mechanism, the overhead may be reduced because allocations could be optimized to be done as a whole, instead of one by one. However, the number of tasks that will be created and scheduled is still proportional to the problem size.

Task nesting could be done using the previously mentioned taskloop. Concretely, it is possible to implement a code that is firstly partitioned using tasks with dependences that contain taskloops with no dependences-in fact, taskloop does not support data dependences yet. Withal, this is very similar to the previously described implementation using pure task nesting.

\section{B. Non-OpenMP related work}

There exist other works, such as StarPU Parallel tasks [14]. A parallel task is a task that can be run in parallel by a set of CPUs, which might sound similar to our proposal of worksharing tasks. Nonetheless, these tasks are like the combination of an OpenMP task with a worksharing construct inside. Thus, it contains an implicit barrier at the end. Moreover, in many cases, only a single parallel task can run at a time. The reason for this is that many environments and libraries they leverage internally do not support concurrent calls to create several parallel regions without nesting.

Intel Cilk presents the cilk_for [15], which is used to parallelize loops. The body of the loop is converted into a function that is called recursively using a divide and conquer strategy for achieving better performance. However, there is a cilk_sync at the end of each iteration. Therefore, synchronization is quite rigid, similarly to OpenMP worksharings.

\section{WORKSHARING TASKS}

In this section, we detail the semantics of our proposal, its constraints and how it fits into the tasking model. Also, we discuss its applicability and utility.

\section{A. Syntax}

We propose a new clause for the task construct. This is the for clause for $\mathrm{C} / \mathrm{C}++$ and the do for Fortran.

A task for-or worksharing task-accepts all the clauses accepted by a regular task except the final clause because task for is always final. Note that being final means that no tasks can be created inside the context of a worksharing task. Additionally, it accepts the

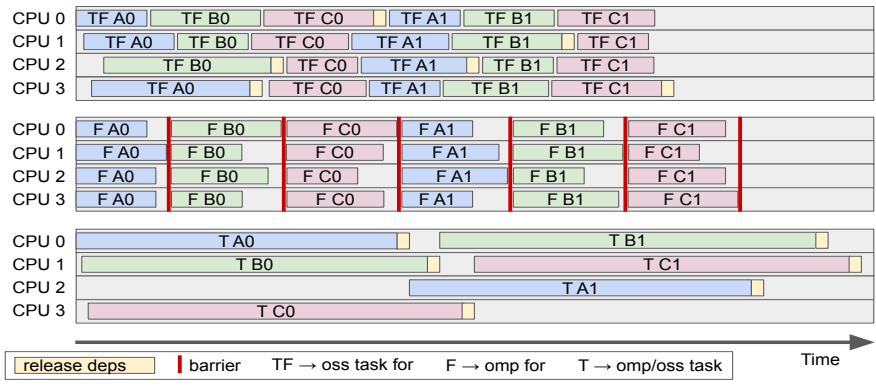

Fig. 2: Illustration of similar execution using OmpSs-2 worksharing tasks and OpenMP worksharings.

chunksize(integer-expr) clause. The integer-expr specified as a chunksize sets the minimum chunk of iterations that each worker is going to execute when it requests work to the worksharing task, except for the last chunk that might contain fewer iterations. If not set, the default value is Tasksize/NumberOfCollaborators, so that each collaborator has at least one chunk to run.

The for clause can only be applied to a task that is immediately succeeded by a loop statement. Codes 3 and 4 contain examples of code using the new clause.

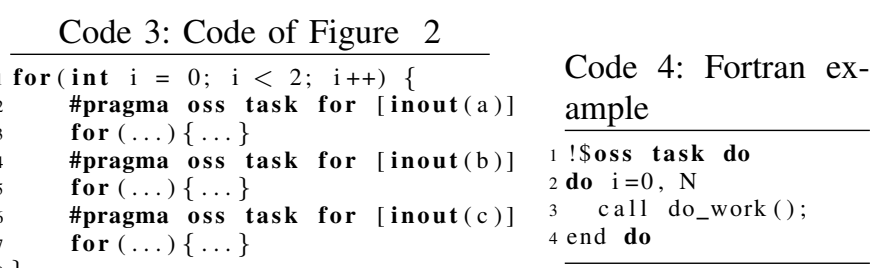
$8\}$

\section{B. Semantics}

A worksharing task behaves like a regular task in almost everything. The main difference is illustrated in Figure 2 whose code is shown in Code 3 Regular tasks are executed entirely by a single worker concurrently, while a task for may be executed collaboratively by several workers, as a worksharing construct. Nevertheless, one can see in Figure 2 that it does not imply any synchronization or barrier at all. A worksharing task is like a regular task in this sense, and the synchronization is done through data dependences or explicit synchronization points. Note that the data dependences of the worksharing tasks are released when the last chunk is finished by the thread that runs that last chunk. This can be seen in Figure 2, represented by the small yellow piece at the end of the last chunk of each worksharing task.

As a worksharing construct, the iteration space of the forloop is partitioned in chunks of chunksize size. The key point is that these chunks do not have the usual overheads associated with a task - such as memory allocation and dependences management. To run a chunk, a thread only needs the boundaries of that chunk and the data environment, much like worksharing constructs. So, in summary, a worksharing task can be run in parallel by multiple threads, better amortizing the task management overheads.

Usually, programmers use coarse granularities to overcome tasking overheads. Using tasks, coarse granularities may limit 
parallelism, causing some resources to be idle as in the bottom part of Figure 2. In contrast, using coarse-grained worksharing tasks, the work is split again into several fine-grained chunks that can be run concurrently by several workers. Thus, preventing resources from becoming idle and maximizing resource utilization, as shown in the top part of Figure 2.

Regarding chunk distribution, a worksharing task is highly flexible. The only guarantee is that work is partitioned in chunks of chunksize size and it is executed at most by $\mathrm{N}$ collaborators of the same team.

A worksharing task creates a worksharing region that is executed by a team of workers. One important property of worksharing regions is illustrated in Figure 2 up to $\mathrm{N}$ threads may collaborate on the completion of the work, but they are not forced to do so. This behavior happens with $T F A O$ and $T F B O$ which are run only by three threads while their team contains four threads. This happens because all the work has been assigned prior to the arrival of the last thread, so the last thread just goes ahead and gets more work.

A further key feature can be observed in Figure 2. CPUs can leave the worksharing region before the actual completion of the whole worksharing task. CPUO finishes its $T F A O$ chunk while CPU1 and CPU3 are still completing their chunks. However, instead of waiting as a regular worksharing does in the middle part of the figure, it moves forward to TF $B O$. In other words, worksharing regions do not contain implicit barriers at the end. This behavior is equivalent to set a nowait clause in OpenMP worksharing constructs.

This feature is especially important because it permits the pipelining of different worksharing regions. This behavior can be observed in Figure 2. For instance, when CPU1 finishes its $T F A O$ chunk, there is no remaining work in $T F A O$. Hence, it leaves that worksharing region and joins $T F B O$. However, TF AO was still in execution by CPU3.

In summary, worksharing tasks implicitly alleviate the effects of a possible load imbalance through the ability of collaborators to leave a worksharing region when there is no remaining work. Thus, threads can just go forward and get more work instead of becoming idle waiting at a barrier. Worksharing tasks also palliate the granularity issues by allowing the use of coarse granularities that are partitioned anew at an additional level of parallelism. So, task management overheads are minimized, and resource utilization is maximized.

\section{Integration in OmpSs-2}

The concept of worksharing task is completely integrated into the model since at all levels it is like a task, except that it may be executed by several workers instead of by a single one. For that reason, it can interact with regular tasks without further problem using regular mechanisms: data dependences and explicit synchronization points regarding synchronization; and data-sharings for managing how the data is shared across different pieces of work.

\section{Applicability}

Worksharing tasks applicability is as wide as OpenMP worksharings. If the iterations in a loop are independent,

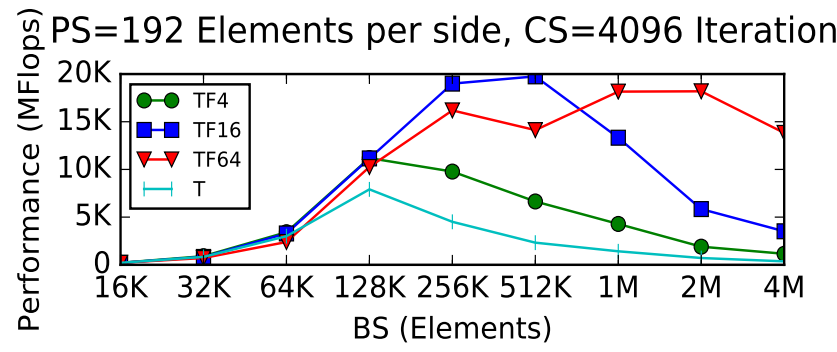

Fig. 3: Comparison of HPCCG benchmark using region dependences with regular tasks and worksharing tasks.

then worksharing tasks can be applied. Worksharing tasks are especially useful to deal with applications containing multiple kernels especially if those present different patterns (regular/irregular). Worksharing tasks enables users to program using a pure data-flow model while efficiently exploiting finegrained loop parallelism. Some benchmarks that we expect to benefit from worksharing tasks and we are looking at, or plan to do so in the future are: Strassen, XSBench, SparseLU, MiniTri, Jacobi and miniAMR, to name a few.

\section{E. Utility}

Worksharing tasks mitigate or solve the problems presented in Section II. Firstly, worksharing tasks enlarge the set of granularities that deliver good performance. In scenarios where only a few tasks are created and if these are not enough to keep all the resources busy, the use of worksharing tasks mitigate the lack of parallelism. Thus, providing several extra granularities that still work well compared to regular tasks, overall, easing the granularity choice.

Furthermore, as we already discussed in Section II. there are scenarios when a good granularity does not exist and developers incur either on overhead or lack of parallelism. For that scenarios, worksharing tasks are especially useful because developers can reduce overhead by setting coarser granularities, without fearing a lack of parallelism. Given that worksharing tasks split the work among a whole team of collaborators, the total number of tasks required to keep all the resources busy is reduced from the total number of cores to the total number of teams. Hence, offering a solution to scenarios where tasks are unable to perform well.

Finally, since worksharing tasks are able to reduce the number of tasks by making them coarser without any significant performance loss, runtime libraries can develop sophisticated mechanisms to deal with task management. One example can be seen in Figure 3, where region dependences are in use. They are not suitable to be used with regular tasks given its low performance. However, they become suitable when combined with worksharing tasks.

\section{IMPLEMENTATION DETAILS}

We have implemented the concept of worksharing tasks in the OmpSs-2 programming model, which relies on the Mercurium source-to-source compiler [16] and the Nanos6 
runtime library [17]. In this section, we detail the extensions performed in both components to support worksharing tasks.

\section{A. Mercurium compiler}

We have extended the Mercurium compiler to support the new for clause applied to the task construct. Though, as this combination of task for can only be applied to loop statements, Mercurium is also in charge of checking so. In the same line, Mercurium also checks that final clause is not applied to a task for since it is not valid.

Given that a worksharing task may be executed by several different threads, each of them should have a correct data environment to avoid possible errors in the computations. Sometimes, this may imply firstprivate or private datasharings. Mercurium has the responsibility of providing a valid data duplication method to the runtime if firstprivate or private data-sharings have been set by the user. Otherwise, the runtime may not know how to duplicate data.

\section{B. Nanos6 runtime library}

Regarding the runtime library, firstly, we have extended the work descriptor of a task to include some extra information that permits splitting and spreading of the work. Basically, we add information about the boundaries of the loop and the chunksize. This information is taken at task creation time.

Then, when the worksharing task becomes ready, it is enqueued as the rest of the tasks. Eventually, the task is scheduled for execution. At this point, worksharing tasks follow a different path from regular tasks. Regular tasks are assigned to a worker thread, and it is in charge of executing the task and release its dependences if any. Worksharing tasks are also initially assigned to a worker thread but, instead of executing the whole task itself, it shares the task with its team.

Currently, in our implementation, the maximum size of the teams is defined at the beginning of the execution and remain unchanged until the end. Moreover, all the teams have the same maximum size. We build teams by grouping together hardware threads that are close in the physical layout.

The way a worksharing task is actually executed also differs from regular tasks. While for regular tasks we simply assign a work descriptor with its respective data environment to a thread and it just runs; worksharing tasks need some further steps. First of all, as several workers may collaborate to do the work, each of them needs its own work descriptor and data environment to avoid interferences caused by the concurrency.

Each CPU has a preallocated extended work descriptor. When this CPU receives a chunk, the preallocated extended work descriptor is filled with the actual information of the chunk that has been assigned. This represents the control information for running its part of the worksharing task.

Regarding the data environment, given that we do not know in advance how many collaborators will be-neither which of them-, each worker triggers the duplication of the data environment in a lazy way. The worker triggers the data duplication when it has received work to do, and it has filled its preallocated work descriptor with the control information.
Using the data duplication method provided by the compiler, the runtime duplicates the data environment and assigns it to the preallocated work descriptor of the thread.

Once a CPU already has the work descriptor and the data environment, it can start running its part of the work. The assignment of work from a worksharing task is done on a first-come-first-serve basis. It is guaranteed that a worker never receives fewer iterations than those specified in the chunksize clause, except the last chunk if there are not enough iterations to complete a chunk. However, it may receive several chunks.

In the current implementation, the chunk scheduling policy is very similar to the guided scheduling policy of OpenMP since the number of assigned chunks is proportional to the number of unassigned chunks divided by the number of collaborators. Note that no matter how many chunks a collaborator receives, it performs the work descriptor filling and the data environment duplication only once per work request. After terminating the assigned chunks, a thread checks if it is the last. If so, the worksharing task has finished all the chunks, and as a result, it has finished as a whole. Data dependences, if any, are released at this moment.

Otherwise, when a worker finishes its assigned chunks but the whole worksharing task has not finished there exist two possibilities: (1) all the work has been assigned and other collaborators are still running; (2) there is still work to be assigned. In (1), the worker that finishes its chunks just leaves the team and tries to get new work. In (2), the worker requests more chunks to the current worksharing task.

It is also important to highlight that assigning chunks to a worker and finishing those chunks imply some overheads that regular tasks do not have. Even though we have tuned our implementation to allow fine-grained chunks, setting an adequate chunksize is important for the proper exploitation of worksharing tasks as shown in Section VI-D Furthermore, the process of requesting work crosses the scheduler path. So, it has some associated locks that may be taken into account when setting the chunksize.

\section{EVALUATION}

In this section, we provide an evaluation of our proposal, as well as a discussion of the results. First of all, we introduce the environments and platforms in which the experiments were conducted. Following, the benchmarks used and the different implementations developed are described. Then, for each experiment, we detail the methodology followed along with the results and discussion about them.

We include three different experiments. The first one is a granularity analysis on a many-core system. The objective is to show how the traditional ways of exploiting parallelism may easily suffer from a lack of parallelism on many-core architectures. The second experiment is a chunksize granularity analysis which aims to stress the importance of an adequate chunksize. Finally, the third experiment is a strong scaling experiment to illustrate some scenarios where the problem size per core prevents setting a good task granularity. 
We wish to remark that OmpSs-2 implements region dependences. In contrast, OpenMP implements discrete dependences. Given that difference, we have introduced a new dependency system that supports discrete dependences in OmpSs-2 to make comparisons fairer.

\section{A. Environment}

The experiments were carried out on two different platforms. The first platform is composed of nodes with 2 sockets Intel Xeon Platinum 8160 2.1GHz 24-core and 96GB of main memory. The second platform is composed of nodes with 1 socket Intel Xeon Phi CPU 7230 1.3GHz 64-core and 96GB of main memory plus $16 \mathrm{~GB}$ of high bandwdith memory.

Regarding the software, we used the Mercurium compiler (v2.3.0), the Nanos6 runtime library, the gcc and gfortran compilers (v7.2.0), and the Intel compilers (v17.0.4).

We would like to highlight that all the experiments have been run using the interleaving policy offered by the numactl command, spreading the data evenly across all the available NUMA nodes, in order to minimize the NUMA effect.

\section{B. Benchmarks}

We have considered four different benchmarks for the evaluation: the High Performance Computing Conjugate Gradient (HPCCG) [18], the matrix multiplication kernel (MATMUL), the N-body simulation and the Stream benchmark [19]. HPCCG and the Stream benchmark were selected as representants of memory-bounded benchmarks while MATMUL and the N-body simulation represent computed-bounded workload.

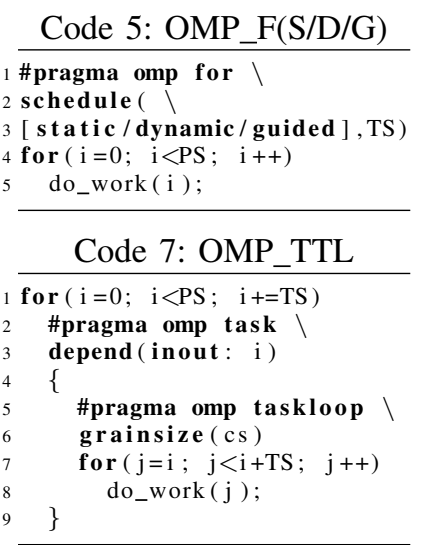

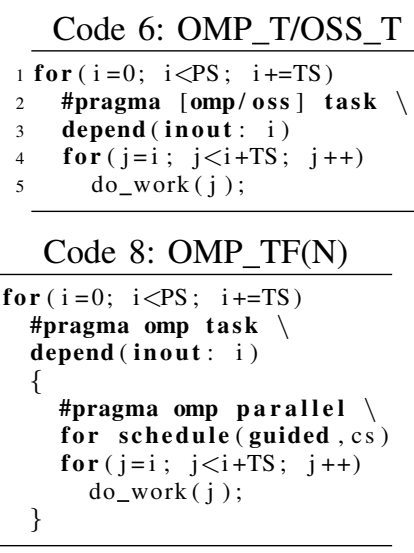

Code 9: OSS_TF(N)

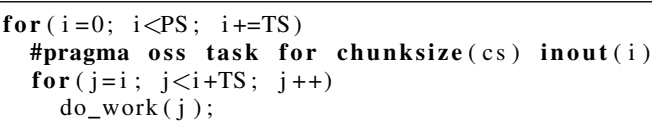

For each of them, we have developed six different versions. Code 5 implements a version using OpenMP parallel for with the static (OMP_F(S)), dynamic (OMP_F(D)) or guided (OMP_F(G)) scheduler. Code 6 shows a version using tasks in both OpenMP and OmpSs-2. This is a blocked version where each task computes a block of TS size. Code 7 is a version using the OpenMP taskloop. However, as taskloops do not accept data dependences, there is a first decomposition using tasks with data dependences. Then, inside each task, the block

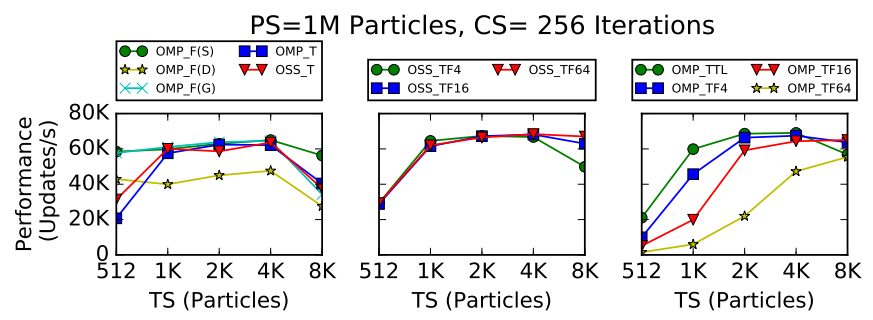

Fig. 4: Granularity chart of different implementations of the $\mathrm{N}$-body simulation.

of TS size is partitioned anew using a taskloop. Code 8 is quite similar to the previous code just replacing the taskloop inside the tasks by a parallel for with guided scheduling, to make it similar to our worksharing tasks. Finally, Code 9 illustrates an implementation done with worksharing tasks.

The $\mathrm{N}$ in codes 8 and 9 indicates the number of threads used in each worksharing construct and the maximum number of collaborators in a worksharing task, respectively.

As a final remark, all the OpenMP implementations have been compiled and run with Intel OpenMP.

\section{Granularity evaluation in many-core architecture}

This subsection is devoted to performing a deep evaluation of our proposal in a many-core architecture such as the Intel KNL. So, the experiments were conducted on the second platform.

In this section, we analyze the behavior of a computebound benchmark, the N-body simulation; and a memorybound benchmark, the Stream benchmark.

In this experiment, we wish to show how the traditional ways of exploiting parallelism-worksharings and taskssuffer from a lack of parallelism when the granularity is coarse. In that scenario, the versions using nested levels of parallelism-OSS_TF, OMP_TTL and OMP_TF_can perform better because they allow higher resource utilization.

The results presented were obtained by averaging the execution times of 5 different runs per version.

1) $\mathrm{N}$-body simulation: Left chart of Figure 4 compares how OMP_T, several OMP_F, and OSS_T versions perform with different granularities. The $\mathrm{x}$-axis determines the size of the blocks. The y-axis represents performance. For the OMP_F version, TS means the chunksize specified in the schedule (static, TS) clause.

OMP_F (S) and OMP_F (G) implementations perform well almost across the whole set of granularities but the last one. This happens because the heaviest computational loop contains as many iterations as the number of blocks. Thus, when the block size is $8 \mathrm{~K}$, there is a lack of parallelism because there is only work for $(1 M / 8 K=128)$ threads, so the other 128 are idle, and performance falls. Note that these versions performs well even when using quite small block sizes, where tasks suffer. This is because worksharing constructs introduce a few overhead in comparison with tasks. Notwithstanding, dynamic scheduler is performing quite badly. The overhead is introduced by the dynamic handling of chunks. It also happens with guided scheduling, but dynamic uses exactly the 

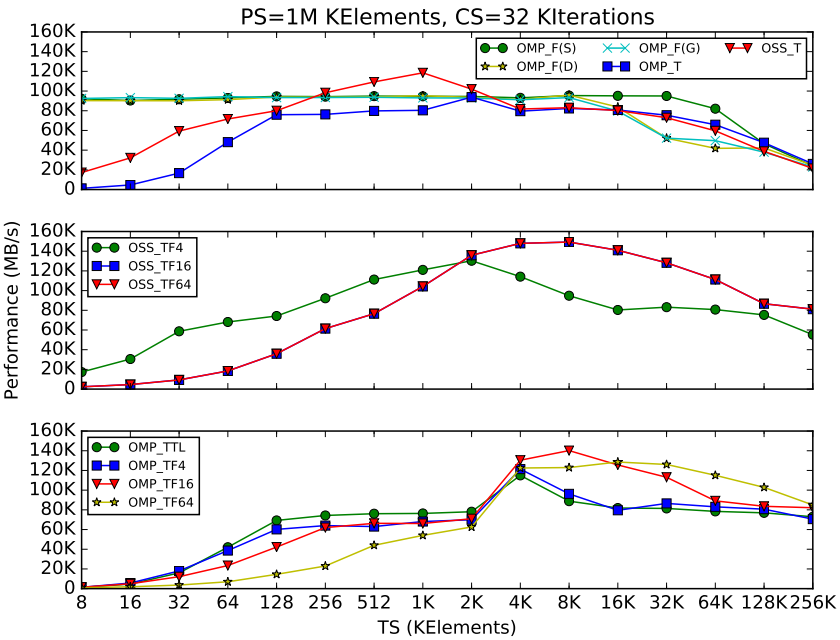

Fig. 5: Granularity chart of different implementations of the Stream benchmark.

chunksize set by the user, while guided uses it as a minimum, and so may get bigger chunks, reducing the overall number of chunks and consequently the overhead.

The OMP_T and OSS_T versions start far from the worksharing because of the overhead introduced by tasks where the granularity is too fine. Then, they get peak performance until the last granularity when the performance falls for the same exact reason than OMP_F: there is not enough parallelism.

The second and third chart of Figure 4 exhibit the results for OSS_TF (N), and OMP_TF (N) and OMP_TTL, respectively. There, one can see an important difference with respect to the previous versions. The difference is that performance does not fall for the biggest granularity when $\mathrm{N}$ is big enough. This means that these implementations are able to prevent the lack of parallelism when the granularities are too coarse. As all these implementations are using a nested level of parallelism, the lack of parallelism in the outer level is alleviated by using the idle resources in the inner level. Consequently, peak performance is maintained for a broader set of granularities than traditional implementations do.

That being said, there are some other interesting points in the second and third charts of Figure 4 Firstly, it is possible to observe divergences among the distinct OpenMP series in the third chart. OMP_TTL adds no extra overhead compared with using only tasks, in the lower granularities. Then, it gives a small boost to the performance in the peak granularities. Finally, for the coarser granularities, it starts falling, but the drop is less pronounced than the drop in the OMP_T version. In contrast, all the OMP_TF versions are introducing extra overhead comparing with OMP_T. Note that this extra overhead becomes bigger as $\mathrm{N}$ grows. However, they are also able to provide a small increase in the peak, like OMP_TTL.

On the other hand, the OSS_TF versions, shown in the second chart of Figure 4, are not introducing further overhead with respect to OSS_T, even with the biggest $\mathrm{N}$, while they are also introducing a small improvement in the peak performance.
2) Stream benchmark: The topmost chart of Figure 5 compares the performance of OMP_T, OMP_E, and OSS_T versions using different granularities. The $\mathrm{x}$-axis determines the size of the blocks. The y-axis represents memory bandwidth. For the OMP_F version, TS means the chunksize specified in the schedule (static,TS) clause.

The main difference between the Stream benchmark and the $\mathrm{N}$-body simulation is the weight of the computation, which is much lighter for the Stream benchmark. Therefore, it needs bigger granularities to hide the overhead of tasks. In the topmost chart of Figure 5 the first granularity in which tasks get good performance is 3072 Kbytes, while for the N-body simulation, it was 44 Kbytes, almost 70x more. Given that we need bigger granularities, it is more likely to end up in a granularity that constraints parallelism.

Looking at the topmost chart of Figure 5, it is interesting to point out that the OMP_F versions performs well even for the lowest granularity, as happened in the N-body simulation, confirming that it introduces very small overhead. All the OMP_F versions perform very similarly except for the biggest granularities. The reason for that remains in a small change in the source code: the version with static scheduler can use the nowait clause. Hence, the OMP_F (S) version only waits once at the end of the four loops while the rest waits four times, one per each loop. So, when granularities are fine, each thread runs several chunks and load balancing problems can be solved by the dynamic/guided scheduler. However, with the biggest granularities, very few chunks, or even a single one, are run by each thread, so load balance problems make a significant difference, happening 4 times against only 1 .

Apart from this, in the same chart is possible to see the tasking versions outperforming all $\mathrm{OMP} \_\mathrm{F}$ versions at some specific granularities. The reason for this is that tasking versions are able to exploit some data locality due to the immediate successor mechanism of the scheduler. With this mechanism, when a task finishes, if some other successor task becomes ready due to the data dependences release of the finished task, the successor is bypassed to the same CPU to exploit data locality. Finally, for the biggest granularities, there is a performance drop in all the versions since there is insufficient parallelism given that few tasks are created. For instance, for the biggest granularity, only $(1 M / 256 K)=4)$ tasks are created, so 252 threads are idle.

The center chart and the bottom chart of Figure 5 exhibit the results for OSS_TF (N), and OMP_TF (N) and OMP_TTL, respectively. Again, like for the N-body case, there exist important dissimilarities comparing these versions with the ones in the topmost chart of Figure 5 . The main one is that the biggest granularities are not falling so much. Again, the reason for this is that the additional level of parallelism introduced in these implementations palliates the lack of parallelism in the outer level. So, we end up having a wider set of granularities reaching good performance.

Interestingly, in the versions shown in the second and third chart of Figure 5 there is a considerable speedup in comparison with its tasking counterparts. For OmpSs-2, second chart, 
OSS_TF 64 gets a $1.25 \mathrm{x}$ against OSS_T. For OpenMP, the third chart, OMP_TF 16 gets a $1.5 \mathrm{x}$ speedup against $\mathrm{OMP} \_\mathrm{T}$. The reason for this is that they are able to better exploit the memory hierarchy. For instance, when the block size is 8 KElements, using $\mathrm{N}=16$, there are at most 16 tasks running concurrently, that means $3 \mathrm{~GB}$. In contrast, tasks imply $\mathrm{N}=1$, so that means 48GB. The high bandwidth memory of the KNL, which is acting as an L3, has 16GB of capacity. Then, for $\mathrm{N}=16$, the whole dataset fits in $\mathrm{L} 3$, while for $\mathrm{N}=1$, it does not.

Unlike with the N-body case, the OSS_TF (N) versions, shown in the second chart of Figure 5, do introduce further overhead with respect to OSS_T when $\mathrm{N}$ starts growing. The same happens with their OpenMP counterparts, shown in the third chart, and in fact, $\mathrm{OMP}_{-} \mathrm{TF}(\mathrm{N})$ versions are introducing much more overhead than OSS_TF (N). Anew, OMP_TTL does not introduce extra overhead comparing to $\mathrm{OMP}_{-} \mathrm{T}$.

Overall, we would like to highlight how the set of granularities achieving peak performance becomes wider as $\mathrm{N}$ increases. This is a consequence of better resource exploitation. When $\mathrm{N}$ is small and TS is big, few tasks are created. If the number of created tasks is smaller than the number of concurrent teams, it is guaranteed that several resources will do nothing because some teams never get a task, hindering performance. When $\mathrm{N}$ grows, there are fewer teams-with many more collaborators-, and so it becomes more difficult for a team to get no tasks. Thus, it is unlikely that any of the resources remain idle. However, as the team size increases, the contention inside it also increases and may threaten performance. Overall, it looks like the best option is to use a big $\mathrm{N}$, but still allow several concurrent teams.

The goal of this experiment is to show that traditional ways of exploiting parallelism suffer from a lack of parallelism when using coarse granularities. We did show that lack of parallelism on traditional approaches. However worksharing tasks still perform well in scenarios where traditional approaches do not. We can conclude that worksharing tasks offer a wider range of granularities delivering good performance making granularity choice easier and not so critical, especially when using large teams. From this experiment, we can also conclude that the number of collaborators $(\mathrm{N})$ is important for achieving good performance. Users must take into account several considerations for choosing it. The first one is the number of worksharing tasks. As happens with regular tasks, the best performance is achieved when all the resources are busy. Therefore, if there are many tasks, $\mathrm{N}$ can be lower, since the teams will be still busy. Oppositely, if there are only a few tasks, $\mathrm{N}$ must be bigger, so that the total number of teams is reduced, and they can be occupied with such a low number of tasks. The second one is lock contention. Each team contains a lock which is shared among all the collaborators. Although it is optimized, more collaborators introduce more contention into the lock. Thus, using a single group with all the available cores may result in performance degradation. The last one is hardware layout. We do not recommend going beyond a CPU socket when setting up teams. As a general recommendation, we suggest using one or two teams per socket. In fact, the
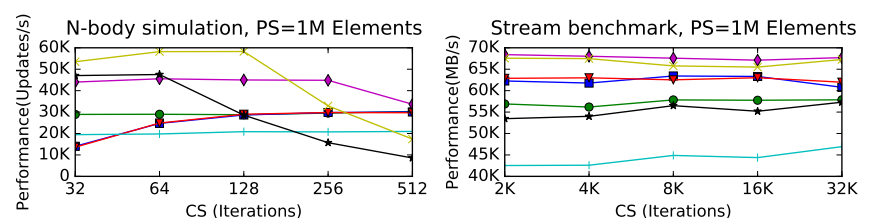

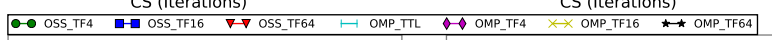

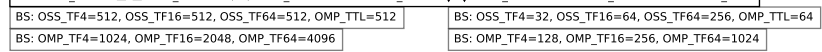

Fig. 6: Chunksize granularity of an N-body simulation and the Stream benchmark.

default value of our implementation is one team per socket.

\section{Chunksize granularity}

The objective of this experiment is to show that the chunksize may affect the performance as much as the task granularity. Thus, it must be considered and adequately tuned.

Figure 6 show an analysis for the N-body simulation and the Stream benchmark of different chunksizes for a fixed problem size. The block size may vary for different versions, but it is the same for all the different chunksizes of a version. In consequence, series cannot be compared against others, only points within the same series can be compared. The block size is a point in the first phase of a typical granularity chart, where tasking overheads still hinder performance.

In the charts, the $\mathrm{x}$-axis stands for chunksize (CS) in number of iterations, while the y-axis shows the performance for the $\mathrm{N}$-body simulation, and the memory bandwidth for the Stream benchmark. The chunksize must be lower or equal than the TS, because a single chunk cannot do more iterations than those in the whole block.

Looking at the right chart of Figure 6 it can be seen that the chunksize does not have any effect on the Stream benchmark. This happens because the limiting resource in this benchmark is the memory. Thus, waiting to acquire some locks, or letting some resources become idle, wasting CPU time, is not so critical like in compute-bound benchmarks.

The chunksize is crucial in the N-body simulation, as can be seen in the left chart of Figure 6 Regarding worksharing tasks, for medium and large values of $\mathrm{N}$, an adequate chunksize provides $+2 \mathrm{x}$ of speedup compared with a bad chunksize. The reason for this is that an excessively small chunksize may imply many more requests to the scheduler, augmenting the contention on the scheduler locks. Making it too large does not affect, because even if there are not enough chunks in a task for to feed all the workers, new work can start.

In contrast, the left chart of Figure 6 shows the opposite behavior for OpenMP. It almost does not matter how small the chunksize is. The OpenMP guided policy assigns chunks dynamically. The actual chunk size is proportional to the number of unassigned chunks divided by the number of threads in the team, with the costraint that it can never be lower than the value set by the user. So, usually, big chunks are assigned at the beginning. Then, they become smaller and smaller until the last iterations where the restriction appears. So, when a user sets a chunksize too small, it only affects a few chunks at the end of the execution, and so it does not 
make a big difference. However, it is affected if the chunksize becomes too big since it cannot feed all the cores and some of them may wait in the barrier until the rest finish.

We have evidenced that chunksize may be important in some applications; while completely nimium in others. As a general recommendation, we suggest using $C S=T S / N$ so that each collaborator in the team has at least one chunk to execute. Nonetheless, having at least one chunk per collaborator is not really important if we have several ready tasks at the same time, because in that case, collaborators can get new tasks. In contrast, when there are only a few ready tasks, it is important to have as many chunks as collaborators or they will probably remain idle. Furthermore, the cost of the computation is also important. Heavier computations can work well with lower chunksizes while lighter computations will require bigger chunksizes to palliate the overheads.

\section{E. Strong scaling}

This experiment consists in fixing a given number of resources and decreasing the problem size, obtaining a smaller problem size per core at each new point of the experiment. The goal is to illustrate that there exist scenarios where the problem size per core prevents the possibility of setting an adequate granularity. In these scenarios, either task management overheads - if the granularity is too fine- or lack of parallelism - if the granularity is too coarse- hinders performance. Thus, by using nested levels of parallelism that allow the use of coarse-grained tasks that are then split into several chunks, performance improves. For this experiment, we have used all the benchmarks presented in Section VI-B.

The results of the experiment are presented in two charts per benchmark, one per platform. In these charts, in the x-axis, there are different problem sizes. The left $y$-axis represents performance while the right y-axis stands for work units per hardware thread. The charts show four different series (bars) for each problem size. Those series are six different implementations, OMP $\_F(S)$, OMP $\_F(D)$, OMP $\_F(G)$, OMP_T, OSS_TF and, finally, the one obtaining best performance between OMP_TF (N) and OMP_TTL. For each of the bars, there is also a circle pointing out the number of work units per hardware thread for that specific configuration. Finally, there is a horizontal line which corresponds to 1 work unit per hardware thread. Thus, it is easy to see when there is at least work for all the resources (above the line) and when there is not (below the line).

For all the versions, we have explored the whole set of combinations for each of the parameters (TS, CS and $\mathrm{N}$ if applicable), and selected the best configuration.

Figure 7 shows the results for the N-body simulation. In the first platform, left chart of Figure 7, all the implementations perform very similarly for the three biggest problem sizes, with all OMP_F versions standing out a bit for the biggest problem size. Then, performance decreases for all the versions. For the biggest problem sizes, all OMP_F versions deliver similar performance. Then, OMP_F (S) outperforms its dynamic and guided counterparts. Dynamic and guided schedulers introduce

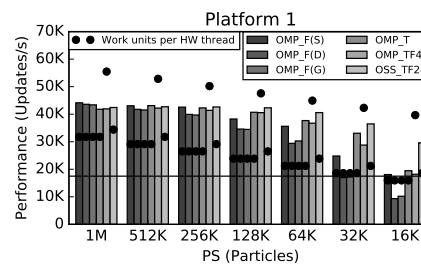

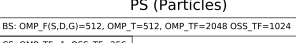

Fig. 7: Strong scaling charts of the N-body simulation.

more overhead than static. They are worth if the application is highly imbalanced, but this is not the case. Hence, they are introducing overhead but not getting any benefit, hurting performance. It is not significant in the biggest problem sizes because the long execution time amortizes the overhead, but it pops up when the execution shortens.

Regarding OMP_F and OMP_T versions, except for the lowest problem size, there is at least one work unit for each hardware thread, so only the lowest granularity has a lack of parallelism. From $32 \mathrm{~K}$ to $128 \mathrm{~K}$, there is a load balancing problem. There are, respectively, 5.33, 2.67, and 1.33 work units per thread for $128 \mathrm{~K}, 64 \mathrm{~K}$, and $32 \mathrm{~K}$. This means that some threads are performing more work than others, and those others are just idle wasting resources.

OMP_TF version has more than enough parallelism when considering both levels of parallelism, but the nested parallel regions are introducing a lot of overhead, and that hurts performance. Additionally, for the lowest problem size, there is not enough parallelism in the first level to feed all the resources, so that even having enough work units when considering combined parallelism, those work units are concentrated in only half of the resources, remaining the rest idle.

In contrast, it can be seen how OSS_TF is able to maintain the performance much better than the other versions, reaching up to a $1.5 \mathrm{x}$ speedup for the lowest size against the best competitor. Note that the problem size is reduced by up to $64 \mathrm{x}$, but OSS_TF performance is still $70 \%$ of the peak performance while the rest are below 50\%. The main reason is that even for the lowest size, we reach high levels of hardware resources ocuppancy thanks to having very few (concretely 2 ) teams with a high amount of CPUs. This allows not only the parallelism to be maximized but also to improve load balancing.

In the second platform, the right chart of Figure 7, the behavior is very similar but accentuated because of the large number of cores available. The performance of OMP_F and OMP_T falls very quickly because of the lack of parallelism. In contrast, both OMP_TF and OSS_TF are able to maintain acceptable performance even when there is not enough parallelism in the first level, thanks to its nested level of parallelism. Nevertheless, OSS_TF outperforms its OpenMP equivalent, becoming the difference between them bigger as the problem size per core decreases. OSS_TF is able to get up to $2 \mathrm{x}$ speedup compared with OMP_TF and more than 5x compared with OMP_T and OMP_F.

Figure 8 shows the results for the MATMUL benchmark.

Regarding the results of the first platform, displayed in the left chart of Figure 8 it is possible to observe one more time the 


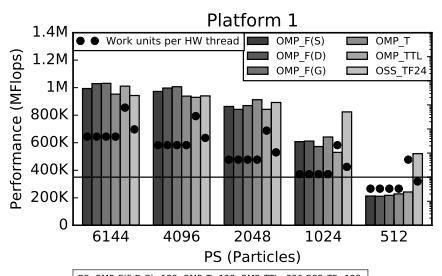

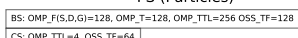

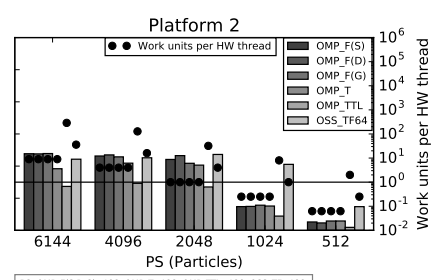

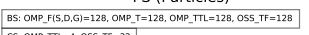

Fig. 8: Strong scaling charts of the matmul benchmark.
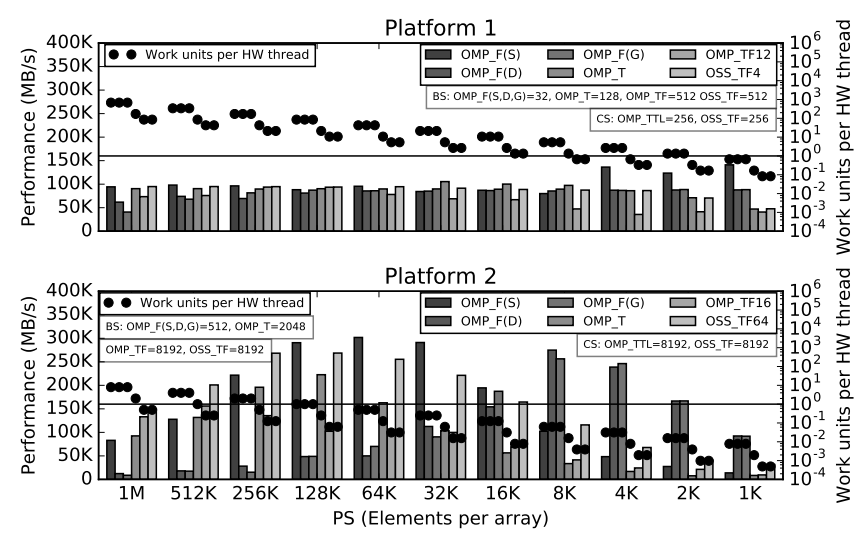

Fig. 9: Strong scaling charts of the Stream benchmark.

performance reduction as the problem size becomes smaller. The reasons are load balancing, like for the N-body simulation, for PS=1024; and the lack of parallelism for PS=512. Yet, OSS_TF keeps performance better than the other versions, achieving up to a $2 \mathrm{x}$ speedup against the best OpenMP version.

The nature of the chart of the second platform, shown in the right chart of Figure 8 , is similar to the previous, but in this case, the main problem is actually the lack of parallelism given the large number of available resources. In this platform, nonetheless, OSS_TF is able to reach up to a $2.7 \mathrm{x}$ speedup versus the best OpenMP version.

The results of the Stream benchmark, available in Figure 9. are different than the previous. In the previous benchmarks, there was a trend where lowering the problem size led to a performance drop, especially in the OpenMP versions.

In the first platform, the topmost chart of Figure 9 , this does not happen, or at least, the drop is not as large. The main reason is that the limiting resource in this benchmark is the memory bandwidth instead of the CPU. Thus, even without using all the resources, peak performance can be achieved. For this reason, decreasing the problem size, leading to a lack of parallelism, is not so important in this benchmark.

That being said, we can see how the OMP_F versions are even increasing its performance, especially $O M P \_F(S)$. The increase stems from the data locality exploitation given that in the lowest sizes, the whole problem or a large part of it fits in the caches. This effect is seen in none of the other versions mainly due to two reasons. The first one is the pollution of the caches caused by the runtime libraries. The second one is that the static scheduling of the OMP_F (S), combined with the nowait clause, allows that a CPU executes the same elements of each loop, maximizing data reuse. In contrast, task-based versions, although they have immediate

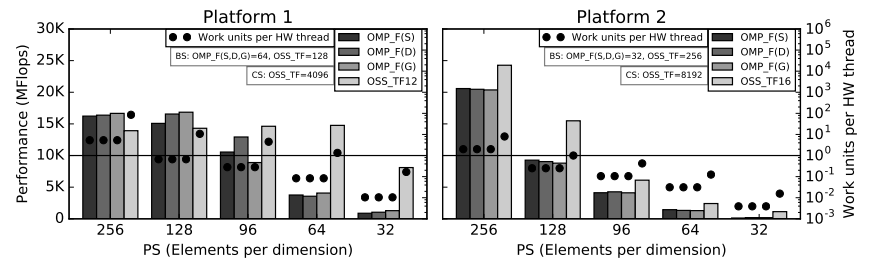

Fig. 10: Strong scaling charts of the HPCCG benchmark.

successor policy which favors locality, is not so perfect as the OMP_F ( S ) one. Regarding the dynamic and guided versions, they need to run the whole loop, iterating over the whole data arrays before moving forward to the next loop, preventing them from any kind of data reuse.

In the second platform, the bottom chart of Figure 9, the effect of insufficient parallelism is notable, like in the previous benchmarks. It is caused by the large number of cores available in this platform, which needs a bigger value of problem size to keep the problem size per core able to perform decently. In this platform, there are four versions that stand out. Again, the reason is data locality. This platform incorporates a $16 \mathrm{~GB}$ high bandwidth memory used as L3. The problem sizes where we get the best performance are those where the whole data set fits in cache while there is enough parallelism.

Figure 10 introduces the results obtained in the HPCCG benchmark. In this benchmark, each chart only contains two series instead of the four mentioned previously. The reason is that the HPCCG benchmark contains several reductions. The Intel OpenMP compiler and runtime we have used do not support task reductions. Therefore, all the versions using tasks (OMP_T, OMP_TF and OMP_TTL) perform poorly.

The results of the first platform are given in the left chart of Figure 10. In that chart, it is possible to see again, like in the previous benchmark, how the performance of all OMP_F versions drops when the problem size per core decreases. In contrast, the performance of OSS_TF remains very similar until the lowest problem size where it finally drops. Despite the drop, OSS_TF gets more than 9x speedup compared to OMP_E for that problem size. The reason for the drop, one more time, is the lack of parallelism. However, it is possible to see some of the circles below the line while the performance is still peak. This happens for the same reason than the Stream benchmark. HPCCG is also memory-bound, so it does not need to occupy all the cores to reach peak performance. There are also some differences depending on the scheduler for the OMP $\_$F versions. Dynamic and guided seems to perform slightly better. The reason is load imbalance.

In the second platform, the right chart of Figure 10, the trends are similar. OMP_F performance is deteriorated by the lack of parallelism. However, the OSS_TF performance in this platform, falls faster because there are many more resources available, and even OSS_TF is not able to exploit enough of them when the problem size is reduced. Still, OSS_TF outperforms OMP_F by up to $1.65 \mathrm{x}$.

We have demonstrated that when performing strong scaling experiments we can easily get into scenarios where the problem size per core prevents traditional ways of exploiting par- 
allelism to get good performance. At the same time, we have shown how worksharing tasks mitigate the lack of parallelism issue being able to perform well across several benchmarks on two different platforms, even in scenarios where traditional approaches suffer.

\section{CONCLUSIONS}

In this paper, we propose a new concept called worksharing tasks that leverage the flexibility of tasks and the efficiency of worksharing techniques to exploit fine-grained loop parallelism. Our proposal introduces the new for clause-do clause in Fortran-to the task construct.

A worksharing task is like a regular task that encompasses a for loop. The key difference is that this for loop can be run by several workers using worksharing techniques that have been adapted to avoid any fork-join synchronization to preserve the fine-grained data-flow execution model of regular tasks.

In general, task-based programming models require at least one task per core to achieve the best performance. This fact provides an upper bound on the task granularity, which proportionally increases with the problem size but proportionally decreases with the number of cores. Thus, a small problem size combined with a large number of cores limits task granularity and impacts performance.

Using too fine-grained tasks, the overheads related to task management hinder performance; while using too coarsegrained tasks, the number of tasks is not enough to fully exploit all cores. The lower bound of task granularity that reaches peak performance is determined by the efficiency of the runtime system to handle tasks, while the upper bound of task granularity is determined by the problem size per core.

Worksharing tasks overcome the requirement of one task per core to achieve high resource utilization by allowing a small number of coarse-grained worksharing tasks that are partitioned into several fine-grained chunks. Worksharing tasks allow us to increase task granularity up to $64 \mathrm{x}$ without limiting the available parallelism. Hence, a small number of worksharing tasks can efficiently exploit a many-core processor.

Our evaluation shows that worksharing tasks not only outperform traditional tasks and worksharing techniques, but also advanced combinations of both techniques. Worksharing tasks get up to $9 \mathrm{x}$ speedup against the most performant OpenMP alternative in some scenarios. Moreover, the use of worksharing tasks increases the range of granularities that reach peak performance. Finally, our proposal does not add any coding complexity over the traditional task-based implementation.

\section{FUTURE WORK}

We plan to investigate additional scheduling policies to distribute loop iterations across workers, as well as dynamic composition of the teams that execute a worksharing task, with the goal of improving the flexibility and efficiency of this mechanism.

Also, we intend to explore the interaction between the OpenMP taskloop and our worksharing tasks. Currently, taskloop distributes a loop into tasks. Given that worksharing tasks are essentially tasks, this should naturally work.

\section{ACKNOWLEDGMENT}

This work is supported by the Spanish Ministerio de Ciencia, Innovación y Universidades (TIN2015-65316-P), by the Generalitat de Catalunya (2014-SGR-1051) and by the European Union's Seventh Framework Programme (FP7/20072013) and the H2020 funding framework under grant agreement no. H2020-FETHPC-754304 (DEEP-EST).

\section{REFERENCES}

[1] OpenMP Architecture Review Board, "OpenMP Application Programming Interface," November 2018, accessed: 2019-0324. [Online]. Available: https://www.openmp.org/wp-content/uploads/ OpenMP-API-Specification-5.0.pdf

[2] J. M. Perez, V. Beltran, J. Labarta, and E. Ayguadé, "Improving the integration of task nesting and dependencies in openmp," in 2017 IEEE International Parallel and Distributed Processing Symposium (IPDPS). IEEE, 2017, pp. 809-818.

[3] E. Ayguade, R. M. Badia, D. Cabrera, A. Duran, M. Gonzalez, F. Igual, D. Jimenez, J. Labarta, X. Martorell, R. Mayo et al., "A proposal to extend the openmp tasking model for heterogeneous architectures," in International Workshop on OpenMP. Springer, 2009, pp. 154-167.

[4] A. Duran, E. Ayguadé, R. M. Badia, J. Labarta, L. Martinell, X. Martorell, and J. Planas, "Ompss: a proposal for programming heterogeneous multi-core architectures," Parallel processing letters, vol. 21, no. 02, pp. 173-193, 2011.

[5] C. Augonnet, S. Thibault, R. Namyst, and P.-A. Wacrenier, "Starpu: a unified platform for task scheduling on heterogeneous multicore architectures," Concurrency and Computation: Practice and Experience, vol. 23, no. 2, pp. 187-198, 2011.

[6] K. Sala, X. Teruel, J. M. Perez, A. Pena, V. Beltran, and J. Labarta, "Integrating blocking and non-blocking mpi primitives with task-based programming models," Parallel Computing, 122018.

[7] Barcelona Supercomputing Center, "OmpSs-2 Programming Model," accessed: 2019-03-24. [Online]. Available: https://pm.bsc.es/ompss-2

[8] T. Harris and S. Kaestle, "Callisto-rts: fine-grain parallel loops," in 2015 $\{$ USENIX\} Annual Technical Conference ( $\{$ USENIX $\}\{A T C\} 15), 2015$, pp. 45-56.

[9] A. Navarro, S. Mateo, J. M. Perez, V. Beltran, and E. Ayguadé, "Adaptive and architecture-independent task granularity for recursive applications," in International Workshop on OpenMP. Springer, 2017, pp. 169-182.

[10] H. Dursun, K.-I. Nomura, L. Peng, R. Seymour, W. Wang, R. K. Kalia, A. Nakano, and P. Vashishta, "A multilevel parallelization framework for high-order stencil computations," in European Conference on Parallel Processing. Springer, 2009, pp. 642-653.

[11] S. Chatterjee, S. Tasirlar, Z. Budimlic, V. Cave, M. Chabbi, M. Grossman, V. Sarkar, and Y. Yan, "Integrating asynchronous task parallelism with mpi," in 2013 IEEE 27th International Symposium on Parallel and Distributed Processing. IEEE, 2013, pp. 712-725.

[12] R. Rabenseifner, G. Hager, and G. Jost, "Hybrid mpi/openmp parallel programming on clusters of multi-core smp nodes," in 2009 17th Euromicro international conference on parallel, distributed and networkbased processing. IEEE, 2009, pp. 427-436.

[13] R. Nanjegowda, O. Hernandez, B. Chapman, and H. H. Jin, "Scalability evaluation of barrier algorithms for openmp," in International Workshop on OpenMP. Springer, 2009, pp. 42-52.

[14] Université de Bordeaux, CNRS and Inria, "StarPU Handbook Parallel Tasks," April 2019, accessed: 2019-04-04. [Online]. Available: http://starpu.gforge.inria.fr/doc/html/TasksInStarPU.html\#ParallelTasks

[15] Intel, "Intel C++ Compiler 19.0 Developer Guide and Reference," accessed: 2019-03-24. [Online]. Available: https://software.intel.com/ en-us/cpp-compiler-developer-guide-and-reference-cilk-for

[16] Barcelona Supercomputing Center, "Mercurium Compiler," accessed: 2019-03-24. [Online]. Available: https://github.com/bsc-pm/mcxx

[17] — , "Nanos6 Runtime," accessed: 2019-03-24. [Online]. Available: https://github.com/bsc-pm/nanos6

[18] Michael A. Heroux, "High Performance Computing Conjugate Gradients: The original Mantevo miniapp," accessed: 2019-03-24. [Online]. Available: https://github.com/Mantevo/HPCCG

[19] University of Tennessee, "HPC Challenge Benchmark," accessed: 2019-03-24. [Online]. Available: http://icl.cs.utk.edu/hpcc/ 